\title{
Customization, transparency and proximity: a user- centered content strategy applied to the design of a virtual library website
}

\author{
Mireia Leg $^{1}$, Mireia Pérez Cervera ${ }^{2}$, Pablo Rebaque-Rivas ${ }^{1}$ \\ ${ }^{1}$ Universitat Oberta de Catalunya, Community Initiatives, Barcelona, Spain \\ \{mleg, prebaque\}@uoc.edu \\ ${ }^{2}$ Universitat Oberta de Catalunya, Virtual Library, Barcelona, Spain \\ mperezcerver@uoc.edu
}

\begin{abstract}
We present a case study of the redesign of the organizational presentation and content of the Virtual Library website at the Universitat Oberta de Catalunya (Open University of Catalonia, UOC), based on a user-centered design strategy. The aim of the redesign was to provide users with more intuitive, usable and understandable content (textual content, resources and services) by implementing criteria of customization, transparency and proximity. The study also presents a selection of best practices for applying these criteria to the design of other library websites.
\end{abstract}

Keywords: usability, findability, user-centered design, library websites, academic libraries, best practices, content strategy, customization

\section{Introduction}

Libraries increasingly need to use websites to transmit information and to present their resources and services. This has created a growing awareness of the importance of improving the user experience and raising levels of user satisfaction. As such, there is a growing body of work on the application of user-centered design (UCD) strategies in the creation of library websites [1, 2, 3, 4].

This phenomenon is particularly relevant in the case of virtual libraries, whose websites are the sole means of interaction with users. One such example is the Virtual Library of the Universitat Oberta de Catalunya (Open University of Catalonia, UOC), "the first university in the world [...] that has been built in its entirety from the start on the internet” [5].

adfa, p. 1, 2014

(C) Springer-Verlag Berlin Heidelberg 2014 
In this study we present the successful application of a user-centered design perspective to reconfigure the content structure and information architecture of the Virtual Library website, offering users more intuitive, usable and understandable content (textual content, resources and services) through enhanced customization, transparency and proximity.

The literature makes extensive reference to the usability $[1,2,3,4,6,7,8,9,10$, $11]$, findability [1,9] and architecture of the information on academic library websites $[3,4,8]$. However, we believe that a focus on customization, transparency and proximity gives our case study an extra degree of detail and added value and can serve as a guide to best practice for other institutions looking to implement the same criteria.

\section{Methodology}

User-centered design refers to a philosophy in which the limitations of the end-user of a product are the focus of each stage of the design process [12]. The broad range of techniques employed at each stage in the UCD process gives the necessary flexibility to adapt to specific requirements and resource availability.

The first months in the design stage of the Virtual Library website were used to gather data on the general requirements. We carried out a benchmarking analysis of major public and university library websites from around the world and identified the key aspects of each, analysing the different solutions adopted for elements such as the home page, navigation, presentation of and access to information, user support, services and functionalities, content and language.

To ensure that we would cater satisfactorily for the needs of our different user profiles, we organized a series of focus groups and interviews with Virtual Library staff, researchers, lecturers and students. To complete the requirements analysis, we also conducted a full study of user consultations and problems reported over the preceding year. The information gathering phase generated a number of ideas for new contents and services and identified the principal information architecture requirements for the redesigned site. To assess the ideal information architecture, we created a map of the site, a data model, and a high-fidelity prototype [13] containing the wireframes of the different library sites examined.

Two usability evaluations of the redesigned site were performed. In both cases, a user test methodology was applied, and a sample of four users was selected for each potential user profile (students, teaching staff, researchers, etc.). The tests comprised a series of questions about the user's profile and pattern of use of the website, a selection of tasks to evaluate effectiveness and satisfaction, and open questions to obtain more detailed feedback. 
The first test was conducted with the website at approximately $90 \%$ completion and consisted of a global analysis of the design (graphic design, navigation and content structure, content findability, usability and intuitiveness, suitability of tags, etc.). The second test was conducted six months after the launch of the beta version and was used for a detailed usability analysis of some of the most critical sections and functionalities of the website.

Throughout the study period, we monitored and analysed consultations and problems reported by users and examined the usage statistics of the site as a whole and of individual tools and resources.

\section{$3 \quad$ Results and actions}

The results obtained via the different analysis and evaluation methods provided a complete picture of user behaviour and identified the main challenges and problems that the Virtual Library website must address. Notably, the results revealed that users were unaware of many of the resources, tools and services on offer and felt confused by the abundance of tools for accessing information and the sheer volume of information available.

To address these issues, we opted for a clear, uncluttered design; a clear content structure providing concise, contextualized information; and a higher degree of customization, transparency and proximity to users.

For example, to improve the clarity of textual information we used concise, coherently structured texts, written in a familiar style (using the second person singular, avoiding technical terms). Textual information was further clarified by short accompanying videos.

To transmit the values of quality and transparency that form the basis of the redesign, we chose to provide more comprehensive explanations of processes and services, clearly stating the users' rights and duties, outlining the Library's commitment to service, and drafting a series of regulations applicable to all services.

To improve proximity, information on services (and some content types) was expanded to include the names and photographs of the staff responsible, adding a personal element to what is otherwise an entirely virtual service and encouraging feedback between staff and users.

To improve access to different content types, the site was restructured to reflect user needs (article search, database search, take out a loan, obtain an e-document, etc.) rather than the tools that the Library offers (catalogue, link resolver, discovery tool, metasearch engine, etc.). We also introduced a single search box in a prominent posi- 
tion on the every page that offers a simple, centralized means of accessing any resource. This new functionality runs on the discovery tool Summon1, which can be modified to provide access to resources and contents not originally linked to its unified index, making it highly effective.

The information architecture was customized to display content matching each user profile (students, teaching staff, researchers, etc.), enabling every user to clearly identify the services and resources $\mathrm{s} / \mathrm{he}$ can access and the corresponding terms of use. In addition, to guarantee the necessary visibility and transparency, the information for each profile is now made available to all users.

\section{Conclusions}

A new website is a significant change and a considerable undertaking for everyone involved: the architecture must be designed and developed, staff must learn to use a new content management system, and all users will need time to become familiar with the new site. It is important to be aware of these issues and adopt a responsible approach to make the most of the change. At the UOC, the redesign was taken as an opportunity to implement a new approach focused entirely on users and their varying needs. The UCD philosophy was fundamental in achieving this and has been integrated into the working strategy of the entire Virtual Library team.

As is made clear in the Results section, user satisfaction is tied to the concepts of clarity, simplicity, content structure and contextualization, customization, transparency and proximity. These concepts are key factors in the overall usability of the website, enabling users to find and view the desired content in as straightforward a manner as possible. Given that the potential users of a university library present a broad range of needs and varying degrees of knowledge and information skills, designing a website that caters for them all is no easy task. The concept of customization is therefore paramount.

Feedback from users during the information gathering phase made frequent reference to customization. Users are clearly interested in far more advanced customization and would like to be offered content that meets their specific requirements as individuals rather than as members of a particular profile group. To reinforce the point, many users referred to the recommendation function employed by Amazon.

Future work will continue to focus on the customization of the site content. Forthcoming developments will include the creation of shortcuts to customized content for

\footnotetext{
${ }^{1}$ Summon, from the firm Serials Solutions, "is built around a single, unified index that returns a single, unified set of results-allowing users to effectively search and navigate across almost all of the library's resources" (Source: Serials Solutions website. http://www.serialssolutions.com/en/services/summon).
} 
each user profile and the launch of the new "My library" section, where users will be able to manage their various forms of interaction with the Library (doubts and queries, reporting problems, loans, service requests, etc.). We will also continue our efforts to improve access to the digital collection and to increase the transparency and usability of other content and resource management tools offered by the Virtual Library and UOC, as this is a critical part of the user experience.

\section{$5 \quad$ Best practices}

We present a selection of best practices that may of use to other institutions when designing a virtual library website:

- Customize content. Display the content available to and required by each user, adapting the browsing experience to specific profiles and information needs.

- Conduct a detailed study of potential site users (information requirements, likely uses of information, behaviour, habits, etc.). This enables information to be organized in a way that reflects specific needs, designing processes that mirror the logic of different patterns of use.

- Provide content findability solutions adapted to the different user profiles and levels of information skills.

- Ensure that texts are concise and coherently structured and written in a familiar style, making information on the site easier to read and understand.

- Prioritize the quality and transparency of processes and services. This ensures that the rights and duties of each party (user and provider) are clearly defined and establishes a trust relationship between the two.

- Devirtualize the service. Telling users about the team behind a website, service or project creates a trust relationship with the user and encourages mutual feedback.

\section{References}

1. Fox, R., Fox, R.: Weaving the digital library web. OCLC Systems \& Services, 24(1): 8-17 (2008)

2. Newell, P.A. et al. Collaborating for Change: Leveraging Campus Partnerships to Create a User-Centered Library Website. Internet Reference Services Quarterly, 18(3-4):227-246 (2013) 
3. Jiao Guo, Pei Yan.: User-centered information architecture of University Library Website. In: 3rd International Conference on Computer Research and Development, (2): 370-372. IEEE (2011)

4. Ward, J. L. Web site redesign: the University of Washington Libraries' experience. OCLC Systems \& Services, 22(3):207-216 (2006)

5. UOC, The leading online University (sub ENG). Universitat Oberta de Catalunya. [Video] http://youtu.be/O6eQn-PxS3k

6. Kim, Y.-M. Users’ perceptions of university library websites: A unifying view. Library \& Information Science Research, 33(1): 63-72( 2011)

7. Mellone, J. T., Williams, D. J. Applying best practices in web site redesign: the Queens College Libraries experience. OCLC Systems \& Services, 26(3): 177197 (2010)

8. Gullikson, S., Blades, R., Bragdon, M., McKibbon, S., Sparling, M., \& Toms, E. G. The impact of information architecture on academic web site usability. The Electronic Library, 17(5): 293-304 (1999)

9. Blakiston, R. Developing a Content Strategy for an Academic Library Website. Journal of Electronic Resources Librarianship, 25(3):175-191 (2013)

10. Aharony, N. An analysis of American academic libraries\&apos; websites: 2000-2010. Electronic Library, The, 30(6):764-776 (2012)

11. Becker, D. A., \& Yannotta, L. Modeling a library website redesign process: developing a user-centered website through usability testing. Information Technology and Libraries, 32(1): 6 (2013)

12. Garreta-Domingo, M. ; Mor, E. User Centered Design in e-Learning 